\title{
STAT3 nuclear egress requires exportin 7 via engaging lysine acetylation
}

\begin{abstract}
Nucleocytoplasmic shuttling of signaling molecules is crucial for regulating their activity. Regulation of signal transducer and activator of transcription 3 (STAT3) is critical for normal physiology while STAT3 dysregulation underlies many diseases such as cancer. However, the mechanism(s) underlying STAT3 nuclear egress remains unclear. Here, we show that exportin 7 is critical for STAT3 nuclear egress. Lysine acetylation at K685, frequently found in tumors and tumor cell lines, mediates STAT3 engagement with exportin 7. Blocking acetylation through drug administration or mutation of lysine K685 disrupted STAT3's physical interaction with exportin 7, leading to STAT3 nuclear retention. Inhibition of STAT3 lysine acetylation significantly altered the functional localization of exportin 7 from the cell cytoplasm toward the nucleus which can be reversed by treating tumor with a lysine-acetylated peptide spanning STAT3 K685. Taken together, our results have identified exportin 7 as an essential karyopherin for STAT3 nucleocytoplasmic shuttling.
\end{abstract}

Keywords: stat3, exportin 7, nucleocytoplasmic shuttling
Volume I Issue I - 2014

\author{
Andreas Herrmann,' Sergey Nachaev,' \\ Christoph Lahtz,' Heehyoung Lee,' Anne \\ Schroeder, ${ }^{2}$ Brian Armstrong, ${ }^{3}$ Claudia \\ Kowolik, ${ }^{2}$ Marcin Kortylewski,' Richard \\ Jove, ${ }^{2} \mathrm{Hua} \mathrm{Yu}^{\prime}$ \\ 'Department of Cancer Immunotherapeutics \& Tumor \\ Immunology, USA \\ ${ }^{2}$ Department of Molecular Medicine, Beckman Research \\ Institute, USA \\ ${ }^{3}$ Department of Neuroscience, Beckman Research Institute, \\ USA
}

\begin{abstract}
Correspondence: Andreas Herrmann, Beckman Research Institute at the Comprehensive Cancer Center City of Hope 1500 E Duarte Road Duarte, CA 91010 USA, Tel +16262564673, Email aherrmann@coh.org

Hua Yu, Department of Cancer Immunotherapeutics \& Tumor Immunology, Duarte, CA 91010, USA, Email hyu@coh.org
\end{abstract}

Received: April 29, 2014 | Published: May 29, 2014
Abbreviations: STAT, signal transducer and activator of transcription; NPC, nuclear pore complex; NES, nuclear export sequences; FPP, fluorescence protease protection

\section{Introduction}

For a signaling molecule to function effectively it is necessary to have precise cellular localization. Consequently, it is critical to understand the regulation of cellular trafficking- both nuclear translocation and egress of signaling molecules. Signal transducer and activator of transcription 3 (STAT3) plays an important role in normal physiology and in several diseases especially cancer. ${ }^{1-4}$ Activation of STAT3 has been suggested to facilitate its nuclear accumulation. ${ }^{5-7}$ Nuclear STAT3 binds to DNA and regulates gene expression. STAT3 then dissociates from DNA and exits the nuclear compartment through the nuclear pore complex (NPC) into the cytoplasm, where it becomes a substrate for reactivation by kinases residing in the cell cytoplasm or at the outer cell membrane. ${ }^{8-10}$ The reactivation is necessary for STAT3 nuclear retranslocation and transcriptional activity.

Nucleocytoplasmic shuttling of transcription factors is tightly regulated by karyopherins. Karyopherins are a super-family of factors comprising importins and exportins responsible for facilitating trafficking of cargoes through the nuclear pore complex, thereby maintaining cargoes' compartmental balance. ${ }^{11}$ While STAT3 nuclear translocation has been shown to be mediated by importin- $\alpha 3$ and importin- $\beta 1,{ }^{12,13}$ the process that facilitates STAT3 nuclear egress remains poorly understood. ${ }^{7}$ Exportins (exportin 1-7), are thought to engage specific cargoes given by recognition of consensus motifs (nuclear export sequences, NES). ${ }^{14}$ Once exportins have shuttled their cargoes through the NPC in a RanGTP- dependent fashion, cargo molecules are thought to be released upon GTP hydrolysation in the cytoplasm. ${ }^{15,16}$ Important previous studies identified exportin 1/Crm1 to be critical for nuclear export of STAT $1,{ }^{17-19}$ emphasizing a NES that has a potential homologue in STAT3. Nevertheless, a more detailed analysis based on site-directed mutagenesis in STAT3 suggested that STAT3 nucleocytoplasmic shuttling may not involve Crm1.7,20 It remains unclear what exportin(s) is critical for STAT3 nuclear egress. In the current study, we investigated STAT3 nuclear egress in cultured cells as well as in tumors in vivo.

\section{Materials and methods}

\section{Mice and cell culture}

For subcutaneous tumor challenge, athymic nude/nude mice (NCI Frederick) were injected s.c. into the flank with $5 \times 10^{6} \mathrm{MEF}^{\text {Stat } 3 \Delta / \Delta}$ cells stably reconstituted with either STAT3 ${ }^{\mathrm{wt}}$-YFP or STAT3 ${ }^{\mathrm{K} 685 \mathrm{R}}$ YFP. Subcutaneously engrafted $\mathrm{MEF}^{\text {Stat } \Delta \Delta \Delta}$ expressing STAT3K685R-YFP were treated after tumors reached $5-7 \mathrm{~mm}$ in diameter with $10 \mu \mathrm{g}$ peptide/mouse delivered by biolipid-carrier (BioPORTER Protein Delivery Reagent, GenLantis, San Diego, CA) according to manufacturer's instructions. Mouse care and experimental procedures with mice were performed under pathogen-free conditions in accordance with established institutional guidance and approved protocols from the Research Animal Care Committees of the City of Hope.

Stat3-deficient mouse embryonal fibroblasts $\left(\mathrm{MEF}^{\text {Stat } 3 \Delta / \Delta}\right.$, kind gift of Dr. Valeria Poli, Molecular Biotechnology Center, University of Torino, Torino, Italy), mouse 3T3/v-Src fibroblasts (gift of Dr. Richard Jove, The Vaccine \& Gene Therapy Institute of Florida, 
Port St. Lucie, FL) and human glioma U251 cells (provided by Dr. Christine Brown, City of Hope, CA) were cultured in DMEM medium (Gibco) supplemented with 10\% FBS (Sigma). Human prostate adenocarcinoma cells PC3, human prostate carcinoma DU145 and human breast adenocarcinoma MDA-MB468 were purchased from ATCC (American Type Culture Collection, Manassas, VA) and grown according to vendor's instructions. Human colon carcinoma HCT cells with STAT3-wt and mutated STAT3-K685R (kind gift of Dr. Zhenghe Wang, Case Western Reserve University, Cleveland) were grown in McCoy's 5A media supplemented with 10\% FBS (Sigma).

\section{Imaging}

Indirect immunoflourescence was carried out as described previously, ${ }^{6}$ staining pY705-STAT3, STAT3, exportin 7, nucleoporin 50 and 153 (Santa Cruz), Hoechst33342 (Sigma), acetylated STAT3-K685 (Cell Signaling Technology), CD31 (BD Biosciences), and Ki67 (abcam). Fluorescence protease protection (FPP) assay and iFLAP analyses were carried out as described previously. ${ }^{8,21}$ Nuclear accumulation of proteins was calculated as described previously. ${ }^{22}$ The DuoLink ${ }^{\circledR}$ assay to localize interacting proteins was performed according to manufacturer's instructions (Olink Bioscience, Goteborg, Sweden) using antibodies against Stat3 (Stat3 C-20, Santa Cruz) and exportin 7 (Acris). For control, a Stat3 blocking peptide (C-20 Stat3 blocking peptide, Santa Cruz) was added. Tissue array was purchased from BioMax. All specimens were analyzed by confocal microscopy (Zeiss LSM510).

\section{Immunoblotting and immunoprecipitation}

Whole cell lysates were prepared using RIPA lysis buffer containing $50 \mathrm{mM}$ Tris ( $\mathrm{pH} 7.4), 150 \mathrm{mM} \mathrm{NaCl}, 1 \mathrm{mM}$ EDTA, $0.5 \%$ NP-40, $1 \mathrm{mM} \mathrm{NaF}, 15 \%$ glycerol, and $20 \mathrm{mM} \beta$-glycerophosphate. Protease inhibitor cocktail was added fresh to lysis buffer (Mini Protease Inhibitor Cocktail, Roche). Normalized protein amounts were subjected to Western blotting, and immunodetection was performed using antibodies against STAT3, exportin 1, 2, 5, 7 (Santa Cruz), exportin 3 (Millipore), exportin 4 (Epitomics), exportin 6 (Proteintech), pY705-STAT3, acetylated STAT3-K685 (Cell Signaling Technology). Resveratrol was obtained from Cayman Chemical. For co-immunoprecipitation, anti-STAT3, exportin 7, nucleoporin 50 and 153 antibodies (Santa Cruz) were used to label rProtein G agarose beads (Invitrogen), incubated over night with lysates and Western blot detection was performed.

\section{Polymerase chain reaction}

Transcript amplification was determined from RNA purified using RNeasy Kit (QIAGEN). cDNA was synthesized using iScript cDNA Synthesis Kit (Bio-Rad). Real-time PCR was performed using Chromo4 Real-Time Detector (Bio-Rad). Murine and human Gapdh housekeeping genes were used to normalize target gene mRNA levels. Commercially available primers for analyses of STAT3 target genes and XPO7 were obtained from SA Biosciences/QIAGEN (Valencia, $\mathrm{CA})$.

\section{Results and discussion}

\section{Exportin 7 mediates STAT3 nuclear egress via interaction with lysine acetylation}

To identify the karyopherin(s) that regulates STAT3 nuclear export, we carried out STAT3 immunoprecipitation followed by western blotting with indicated exportin antibodies, using protein extracts prepared from human glioma U251 cells. Exportin 7, but not exportins 1-6, was in complex with STAT3, suggesting that exportin 7 might be involved in STAT3 nuclear egress (Figure 1A). Moreover, we determined STAT3 and exportin 7 interaction mainly localized in the cell cytoplasm (Figure 1B). STAT3 interaction with exportin 7 was further shown in tumors in vivo by co-immunoprecipitation using homogenates from human glioma U251 tumors from xenograft mouse models (Figure 1C).
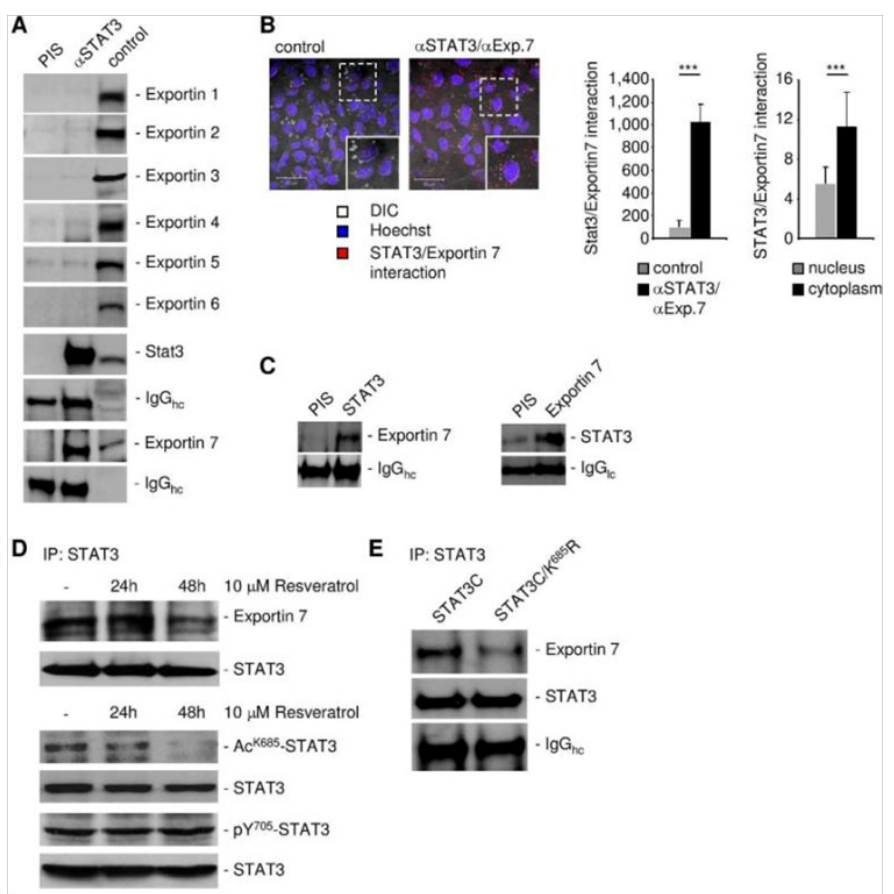

Figure I STAT3 lysine acetylation mediates interaction with exportin 7.

(A) Western blotting following immunoprecipitation of STAT3 showing interaction between STAT3 and exportin 7 using human glioma U25 I cell lysates. Pre-immune serum (PIS) and lysates were included as controls.

(B) Localization of STAT3/exportin 7 interaction (left panels). Glioma U25I was analyzed using anti-STAT3 and anti-exportin 7. For control, a STAT3 blocking peptide was included. Red dot-like fluorescent signals indicate STAT3/exportin 7 interaction. Scale bar, 50 $\mu \mathrm{m}$. STAT3/exportin 7 interaction and subcellular localization of interaction quantified. S.d. shown; significance: ***) $P<0.001$.

(C) STAT3/exportin 7 interaction was validated in vivo by coimmunoprecipitation using tumor homogenates from U25I glioma. PIS served as control.

(D) Western blotting showing effects on STAT3/exportin 7 interaction by resveratrol inhibition of STAT3 lysine acetylation in U25 I glioma.

(E) Western blotting following immuno precipitation showing effects on STAT3/exportin 7 interaction by blocking STAT3 acetylation via sitedirected mutagenesis in constitutively active STAT3C. STAT3C or Stat3C/K685R was expressed in U25I glioma cells.

Since exportin 7 was shown to recognize lysine containing motifs in cargo proteins, ${ }^{23}$ we hypothesized that the main STAT3 acetylation site, lysine $\mathrm{K} 685,{ }^{24}$ might be critical for interaction with exportin 7. Acetylation of STAT3 at K685 was readily detectable in human tumor tissues and cell lines (supplementary material (Supplementary Figure S1). Inhibiting STAT3 acetylation using resveratrol, we showed reduced STAT3/exportin 7 interaction with diminished STAT3 acetylation but not phosphorylation (Figure 1D). Furthermore, 
introducing acetylation mutation in a constitutively activated STAT3 mutant, STAT3C (STAT3C/K685R), resulted in the loss of interaction with exportin 7 (Figure 1E). STAT3 mutation at K685 (STAT3 ${ }^{\mathrm{K} 685 \mathrm{R}}$ ) did not affect STAT3 phosphorylation (supplementary material) (Supplementary Figure S2A) and nuclear translocation upon OSM stimulation (supplementary material) (Supplementary Figure S2B). Cells stably expressing STAT3 ${ }^{\mathrm{K} 685 \mathrm{R}}$, form tumors in vivo at significantly reduced rate compared to STAT3 ${ }^{\mathrm{wt}}$ expressing cells (supplementary material) (Supplementary Figure S3A). Importantly, the reduction of STAT3 ${ }^{\mathrm{K} 685 \mathrm{R}} /$ exportin 7 interaction was validated using tumor homogenates for co-immunoprecipitation experiments (supplementary material) (Supplementary Figure S3B).

Although exportin 1/Crm1 was suggested potentially being involved in STAT3 nuclear export based on the NES that has a homologue in STAT1, so far no direct evidence prove that is the case. Our results indicate that exportin 7 is the main karyopherin critical for STAT3 nucleocytoplasmic shuttling. Furthermore, we show that STAT3 acetylation site is required for exportin 7-mediated STAT3 nuclear export.

\section{STAT3 lysine acetylation is critical for nuclear retention}

To investigate the role of STAT3 acetylation in nuclear retention we assessed STAT3 nuclear egress employing the fluorescence protease protection (FPP) assay ${ }^{21}$ (Figure 2A). Upon distinct permeabilization of the outer cell membrane but not the nuclear envelope, STAT3 ${ }^{\text {wt }}$ underwent rapid nuclear egress while STAT3 ${ }^{\mathrm{K} 685 \mathrm{R}}$ showed an extended nuclear signal (Figure 2B, 2C). Intriguingly, STAT3 ${ }^{\text {wt }}$ interacted with exportin 7 after $2 \mathrm{~h}$ upon ectopic stimulation with a STAT3 activator OSM (Figure 2D). Furthermore, RNAi-based silencing of exportin 7 (XPO7) resulted in decreased induction of STAT3 target genes such as $V E G F$, survivin and $B C_{X L}$ (Figure 2E).

The FPP assay demonstrates that loss of a key acetylation site corrupted efficient STAT3 transport into the cell cytoplasm. The importance of nuclear translocation and export in modulating STAT protein transcriptional activity is well appreciated. ${ }^{7,11,19}$ Data shown in Figure 2 illustrate the point that loss of STAT3 nuclear egress by silencing exportin 7 can affect STAT3 downstream gene expression.

\section{Acetylation is critical for STAT3 nucleocytoplasmic shuttling}

To address STAT3 compartmental turnover, we expressed wildtype STAT3 (STAT3 ${ }^{\mathrm{wt}}$ ) or acetylation deficient STAT3 (STAT3 ${ }^{\mathrm{K} 685 \mathrm{R}}$ ) genetically fused to CFP-YFP in 3T3/v-Src cells. The ability of v-Src to activate STAT3 in the 3 T3 cells has been well documented. ${ }^{4,25-28}$ This allowed us to perform intracatenar fluorescence loss after photobleaching (iFLAP) experiments in living cells. ${ }^{8,29}$ As an internal control for visualization of STAT3 protein mobility and spatiotemporal distribution, a neighboring cell was included in the field of view. Cytoplasmic STAT3 ${ }^{\text {wt }}$-CFP-YFP or STAT3 ${ }^{\text {KR }}$-CFP-YFP was photobleached at $\lambda=514 \mathrm{~nm}$ and followed for subcellular distribution by recording CFP and YFP emissions. Consistent with a previous study, ${ }^{8}$ $\mathrm{STAT}^{\mathrm{wt}}$ migrating from the defined cytoplasmic bleach-ROI entered the cell nucleus as fast as $10 \mathrm{~min}$. However, nuclear migration of acetylation-deficient STAT3 ${ }^{\text {K685R }}$ could not be detected within $15 \mathrm{~min}$ (Figure 3A). Using a more quantitative approach by monitoring nuclear YFP decay under the same experimental setting (Figure 3B), we were able to estimate STAT3 nuclear egress dynamics. While $\mathrm{STAT}^{\mathrm{wt}}$ underwent continuous nuclear decay, STAT3 ${ }^{\mathrm{K} 685 \mathrm{R}}$ fluorescent signals remained constant (Figure 3C).
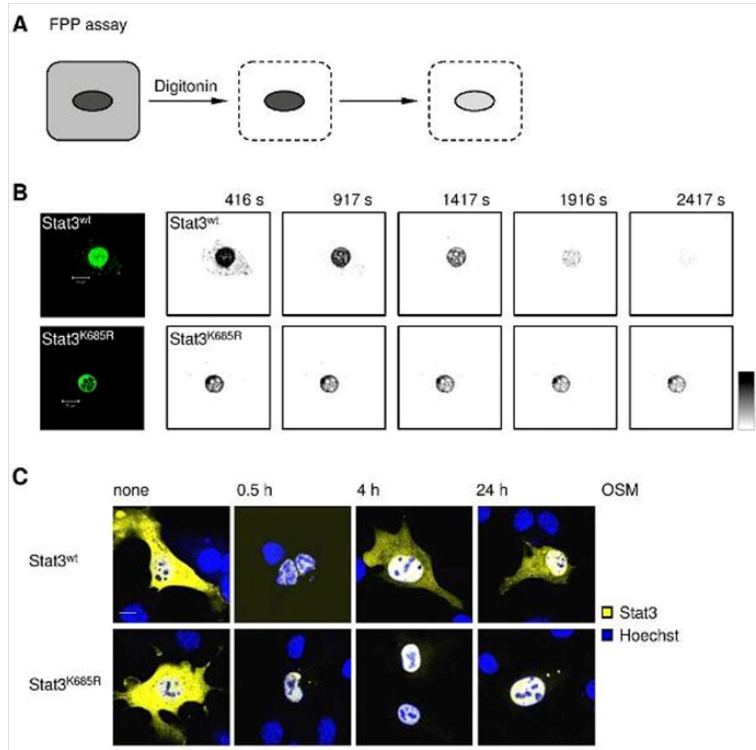

\section{D}
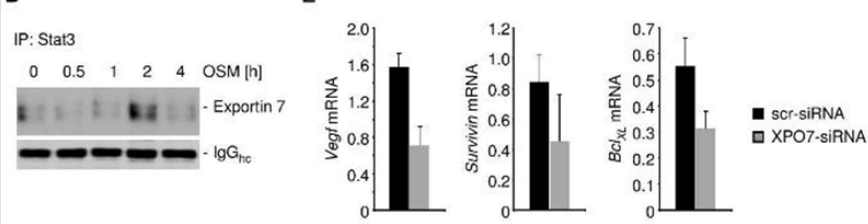

Figure 2 Acetylation-deficient STAT3 undergoes nuclear retention.

(A) FPP assay showing STAT3 nuclear efflux. The cell cytoplasm was cleared from soluble proteins by permeabilizing the outer cell membrane. Decay of nuclear fluorescence indicates efflux/export of nuclear proteins.

(B) Nuclear retention of acetylation deficient STAT3 analyzed in living cells and depicted in intensity-coded-wrong-color-mode. STAT3 ${ }^{\text {wt }}$-GFP and STAT $3^{\mathrm{K} 685 \mathrm{R}}$-GFP expressed in $3 \mathrm{~T} 3 / \mathrm{v}$-Src cells and fluorescent signals were monitored. Scale bar, $10 \mu \mathrm{m}$.

(C) Long-term nuclear retention of acetylation-deficient STAT3 ${ }^{\mathrm{K} 685 \mathrm{R}}$ analyzed in MEF cells upon stimulation with OSM. STAT3 ${ }^{\text {wt }}$-YFP and STAT3 ${ }^{\mathrm{K} 685 \mathrm{R}_{\text {_ }} \text { YFP }}$ were expressed in MEF $\mathrm{Mtat}^{\mathrm{S} \Delta / \Delta}$ cells and treated with $10 \mu \mathrm{g} / \mathrm{ml}$ OSM for times indicated. Cells were analyzed by confocal microscopy. Scale bar, $10 \mu \mathrm{m}$.

(D) Western blotting following immuno precipitation showing OSMinduced STAT3/exportin 7 interaction in MEF cells. STAT3 ${ }^{\text {wt_YFP }}$ expressed in $\mathrm{MEF}^{\mathrm{Stat} 3 \Delta / \Delta}$ cells, treated with OSM and lysates were subjected to co-immunoprecipitation.

(E) RT-PCR demonstrates silencing exportin 7 affects expression of STAT3 target genes. Exportin 7 siRNA was delivered to U25I cells and mRNA levels were determined. A scrambled siRNA served as control.

Nucleoporins of the inner basket of the NPC facilitate the egress of nuclear proteins. ${ }^{16,30,31}$ Nucleoporins 50 and nucleoporin 153 are major components of the NPC representing a check-point for nuclear exit ${ }^{30,31}$ (Figure 3D). We therefore analyzed the interaction of STAT3 $^{\text {wt }}$ and STAT3 ${ }^{\text {K685R }}$ with nucleoporins to assess nuclear egress efficacy. As shown by confocal microscopy, STAT3 ${ }^{\text {wt }}$ colocalized with nucleoporin 50 and 153, resulting in nuclear-envelope-like structures (Figure 3E). However, STAT3 ${ }^{\mathrm{K} 685 \mathrm{R}}$ colocalization with nucleoporins was significantly decreased (Figure $3 \mathrm{~F}$ ), which was concomitant with considerably decreased physical interaction of STAT3 ${ }^{\text {K685R }}$ with nucleoporins compared to STAT3 ${ }^{\text {wt }}$ in vivo (Figure $3 \mathrm{G}$ ) indicating interrupted nucleocytoplasmic shuttling of STAT3 ${ }^{\mathrm{K} 685 \mathrm{R}}$. 

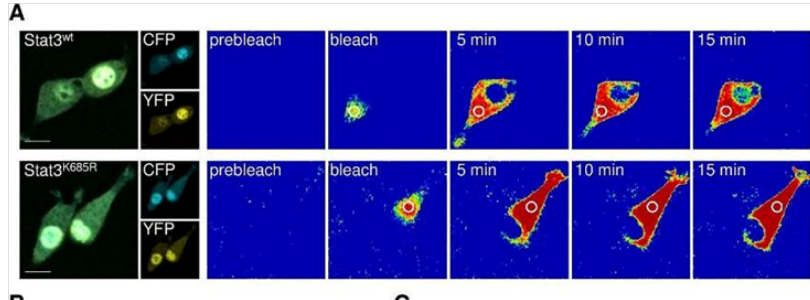

B

C

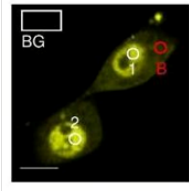

BG - Background-ROI B - Bleach-ROI 1 - detection ROI 1 -
nucleus bleached cell nucleus bleached cell nucleus unbleached cell
nolition 2 -

D
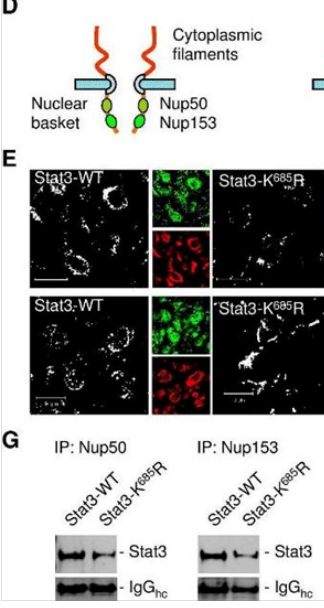
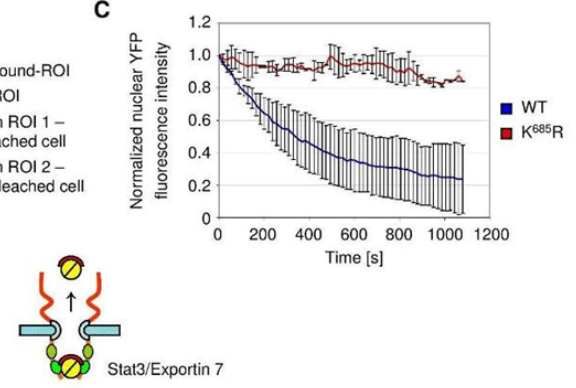

Figure 3 Acetylation is critical for STAT3 nucleocytoplasmic shuttling.

(A) STAT3 compartmental turnover was determined by iFLAP in living cells. STAT3 ${ }^{\text {wt }}$-CFP-YFP and STAT3 ${ }^{\mathrm{K} 685 \mathrm{R}}$-CFP-YFP were expressed in $3 T 3 / v-S r c$ cells. After iterative bleaching, subcellular distribution of STAT3 ${ }^{\text {wt }}$ and STAT3 ${ }^{\mathrm{K} 685 \mathrm{R}}$ was calculated and depicted in wrong-colormode.

(B) For quantification, ROls were employed for determination of compartmental fluorescence.

(C) Compartmental fluorescence indicating STAT3 ${ }^{\text {wt }}$ and STAT3 ${ }^{\text {K685R }}$ nuclear decay upon cytoplasmic bleaching. S.d. shown.

(D) Architecture of the NPC emphasizes nucleoporins 50 and 153 in nuclear egress of cargoes engaged by exportins.

(E) STAT3 colocalization with nucleoporin 50 and 153 in tumors expressing STAT3 ${ }^{\text {wt }}$ or STAT3 ${ }^{\mathrm{K} 685 \mathrm{R}}$. Pixels double-positive for STAT3 and nucleoporins were employed to generate colocalization images. Scale bar, $10 \mu \mathrm{m}$

(F) STAT3/nucleoporin-positive pixels were quantified. S.d. shown; significance: $* * P<0.01$.

(G) STAT3 nuclear exit through NPC was validated in vivo. Homogenates from tumors expressing STAT3 ${ }^{\text {wt }}$ or STAT3 ${ }^{\mathrm{K} 685 \mathrm{R}}$ subjected to coimmunoprecipitation with nucleoporin 50 or 153 and analyzed in Western blotting.

iFLAP illustrates a requirement of acetylation at lysine K685 of STAT3 for effective nuclear cytoplasmic shuttling. Acetylated STAT3 is also needed for STAT3 interaction with nucleoporins 50 and 153, which form check-points for protein nuclear egress. However, these results do not distinguish whether STAT3 directly or indirectly interact with nucleoporins 50 and 153 .

\section{STAT3 acetylation contributes to exportin 7 functional localization}

Exportin 7 was initially identified to recognize protein-motifs containing lysine residues. Once exportin 7 engages its cargo, it is thought to exclude cargoes from nuclei, thereby contributing to compartmental distribution of cargo protein. ${ }^{23}$ Exportin 7 was found in the cell cytoplasm in tumors (Figure 4A, left panel). However, in tumors expressing acetylation-deficient STAT3 ${ }^{\mathrm{K} 685 \mathrm{R}}$, exportin 7 was mainly detected in the cell nucleus when compared to STAT3 ${ }^{\text {wt }}$ expressing tumors (Figure 4A, right panel). Of note, inhibition of STAT3 acetylation did not interfere with exportin 7 mRNA expression levels (Supplementary Figure S4).

To further investigate the role of STAT3 acetylation in subcellular distribution of exportin 7, we engrafted cells stably expressing acetylation-deficient STAT3 ${ }^{\mathrm{K} 685 \mathrm{R}}$ into athymic mice, followed by intratumoral delivery of a lysine-acetylated peptide spanning STAT3 acetylation site (acet.Stat3 pept.) to mimic STAT3 acetylation activity. As a control, an ovalbumin (OVA) peptide was used. The peptides were delivered using a biolipid-carrier system. ${ }^{32}$ While exportin 7 was found in the nucleus of tumor cells, as indicated by colocalization of exportin 7 with the nucleic acid stain Hoechst, delivery of acetylated STAT3 peptide resulted in exportin 7 nuclear exclusion and restoration of the functional localization of exportin 7 (Figure 4B), similar to that of STAT3 ${ }^{\text {wt }}$ expressing tumors (Figure 4A).

A

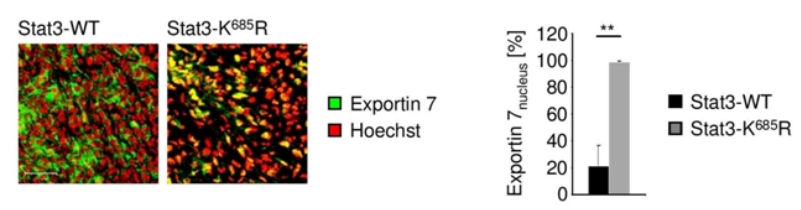

B

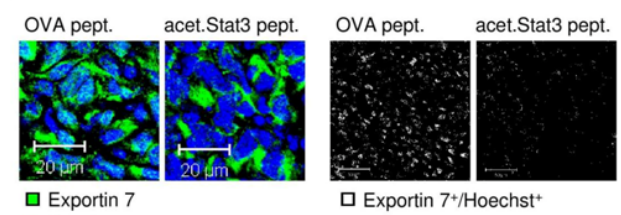

Hoechst

Figure 4 STAT3 acetylation regulates exportin 7 functional localization.

(A) Confocal microscopic analyses indicating exportin 7 localization in $\mathrm{STAT3}^{\mathrm{wt}}$ or STAT3 ${ }^{\mathrm{K} 685 \mathrm{R}}$ expressing tumors. Scale bar, $50 \mu \mathrm{m}$. Distribution of exportin 7 was quantified. S.d. shown; significance: **) $P<0.01$.

(B) Confocal microscopic analysis of exportin 7 localization from tumors expressing STAT3 ${ }^{\mathrm{K} 685 \mathrm{R}}$ treated with STAT3 acetylated peptide spanning K685 (acet.Stat3 pept.) or OVA ${ }^{\text {SIINFEKL }}$ peptide as control. Nuclear exportin 7 were visualized by employing exportin $7 /$ Hoechst-positive pixels. Scale bar, $50 \mu \mathrm{m}$.

The subcellular localization of exportin 7 in tumor tissues was not defined. Our results indicate that it may be mainly located in the cell cytoplasm. Previous pioneering work has demonstrated that exportin 7 recognizes a broad range of cargoes independent of their specific cellular functions. ${ }^{23}$ Based on our current study, it appears that STAT3 acetylation is a substrate for exportin 7, contributing to its nucleocytoplasmic shuttling. This finding is consistent with prior studies showing the importance of lysine residues for high affinity binding by exportin 7 . The number of proteins actively excluded from nucleus is estimated to be very large. ${ }^{23}$ The number of proteins 
potentially acetylated can be very high. However, not all these proteins are regulated by nucleocytoplasmic shuttling. Besides, acetylation site(s) on certain proteins may not be exposed to allow interaction with exportin 7. Perhaps even more important is the fact that acetylation itself can be tightly regulated. In the case of STAT3, acetylation responds to certain cytokines and acetylation of STAT3 is prominent in various human cancers ${ }^{33,34}$ (Supplementary Figure S1). These findings are also supportive of the notion that STAT3 nuclear export is critical for its reactivation, nuclear translocation and transcriptional activity.

\section{Acknowledgements}

This work is funded in part by R01CA122976. Research reported in this publication was supported by the National Cancer Institute of the National Institutes of Health under grant number P30CA033572. The content is solely the responsibility of the authors and does not necessarily represent the official views of the National Institutes of Health.

\section{Conflict of interest}

Andreas Herrmann designed and performed experiments, analyzed data and wrote the manuscript; Sergey Nachaev, Christoph Lahtz, Heehyoung Lee, Anne Schroeder, Brian Armstrong, Claudia Kowolik, Marcin Kortylewski, performed experiments and analyzed data; Richard Jove and Hua Yu wrote the manuscript.

\section{References}

1. Darnell JE. Validating Stat3 in cancer therapy. Nat Med. 2005;11(6):595596.

2. Darnell JE. Transcription factors as targets for cancer therapy. Nat Rev Cancer. 2002;2(10):740-749.

3. Rong Y, Cheng L, Ning H, et al. Wilms' tumor 1 and signal transducers and activators of transcription 3 synergistically promote cell proliferation: a possible mechanism in sporadic Wilms' tumor. Cancer Res. 2006;66(16):8049-8057.

4. Yu H, Jove R. The STATs of cancer-new molecular targets come of age. Nat Rev Cancer. 2004;4(2):97-105.

5. Heinrich PC, Behrmann I, Haan S, et al. Principles of interleukin (IL) 6-type cytokine signaling and its regulation. Biochem J. 2003;374(Pt 1): $1-20$.

6. Herrmann A, Sommer U, Pranada AL, et al. STAT3 is enriched in nuclear bodies. J Cell Sci. 2004;117(Pt 2):339-349.

7. Reich NC, Liu L. Tracking STAT nuclear traffic. Nat Rev Immunol. 2006;6(8):602-612.

8. Herrmann A, Vogt M, Monnigmann M, et al. Nucleocytoplasmic shuttling of persistently activated STAT3. J Cell Sci. 2007;120(Pt 18):3249-3261.

9. Pranada AL, Metz S, Herrmann A, et al. Real time analysis of STAT3 nucleocytoplasmic shuttling. J Biol Chem. 2004;279(15):15114-15123.

10. Yu H, Pardoll D, Jove R. STATs in cancer inflammation and immunity: a leading role for STAT3. Nat Rev Cancer. 2009;9(11):798-809.

11. Xu L, Massague J. Nucleocytoplasmic shuttling of signal transducers Nat Rev Mol Cell Biol. 2004;5(3):209-219.

12. Cimica V, Chen HC, Iyer JK, et al. Dynamics of the STAT3 transcription factor: nuclear import dependent on Ran and importin- $\beta 1$. PLoS One. 2011;6(5):e20188

13. Liu L, McBride KM, Reich NC. STAT3 nuclear import is independent of tyrosine phosphorylation and mediated by importin-alpha3. Proc Natl Acad Sci U S A. 2005;102(23):8150-8155.
14. Guttler T, Gorlich D. Ran-dependent nuclear export mediators: a structural perspective. EMBO J. 2011;30(17):3457-3474.

15. Gorlich D, Mattaj IW. Nucleocytoplasmic transport. Science. 1996;271(5255):1513-1518.

16. Grossman E, Medalia O, Zwerger M. Functional architecture of the nuclear pore complex. Annu Rev Biophys. 2012;41:557-584.

17. McBride KM, Banninger G, McDonald C, et al. Regulated nuclear import of the STAT1 transcription factor by direct binding of importinalpha. EMBO J. 2002;21(7):1754-1763.

18. McBride KM, McDonald C, Reich NC. Nuclear export signal located within theDNA-binding domain of the STAT1transcription factor EMBO J. 2000;19(22):6196-6206.

19. McBride KM, Reich NC. The ins and outs of STAT1 nuclear transport Sci STKE. 2003;2003(195):RE13.

20. Bhattacharya S, Schindler C. Regulation of Stat3 nuclear export. J Clin Invest. 2003;111(4):553-559.

21. Lorenz H, Hailey DW, Wunder C, et al. The fluorescence protease protection (FPP) assay to determine protein localization and membrane topology. Nat Protoc. 2006;1(1):276-279.

22. Hedvat M, Huszar D, Herrmann A, et al. The JAK2 inhibitor AZD1480 potently blocks Stat 3 signaling and oncogenesis in solid tumors. Cancer Cell. 2009;16(6):487-497.

23. Mingot JM, Bohnsack MT, Jakle U, et al. Exportin 7 defines a novel general nuclear export pathway. EMBO J. 2004;23(16):3227-3236.

24. Yuan ZL, Guan YJ, Chatterjee D, et al. Stat3 dimerization regulated by reversible acetylation of a single lysine residue. Science. 2005;307(5707):269-273.

25. Bromberg JF, Horvath CM, Besser D, et al. Stat 3 activation is required for cellular transformation by v-src. Mol Cell Biol. 1998;18(5):25532558.

26. Garcia R, Bowman TL, Niu G, et al. Constitutive activation of Stat3 by the Src and JAK tyrosine kinases participates in growth regulation of human breast carcinoma cells. Oncogene. 2001;20(20):2499-2513.

27. Yu CL, Meyer DJ, Campbell GS, et al. Enhanced DNA-binding activity of a Stat3-related protein in cells transformed by the Src oncoprotein. Science. 1995;269(5220):81-83.

28. Zhang Y, Turkson J, Carter-Su C, et al. Activation of Stat3 in v-Srctransformed fibroblasts requires cooperation of Jak1 kinase activity. $J$ Biol Chem. 2000;275(32):24935-24944.

29. Koster M, Frahm T, Hauser H. Nucleocytoplasmic shuttling revealed by FRAP and FLIP technologies. Curr Opin Biotechnol. 2005;16(1):28-34.

30. Guan T, Kehlenbach RH, Schirmer EC, et al. Nup50 a nucleoplasmically oriented nucleoporin with a role in nuclear protein export. Mol Cell Biol. 2000;20(15):5619-5630.

31. Nakielny S, Shaikh S, Burke B, et al. Nup153 is an M9-containing mobile nucleoporin with a novel Ran-binding domain. EMBO J. 1999;18(7):1982-1995.

32. Zelphati $\mathrm{O}$, Wang $\mathrm{Y}$, Kitada $\mathrm{S}$, et al. Intracellular delivery of proteins with a new lipid-mediated delivery system. J Biol Chem. 2001;276(37):3510335110 .

33. Lee H, Zhang P, Herrmann A, et al. Acetylated STAT3 is crucial for methylation of tumor-suppressor gene promoters and inhibition by resveratrol results in demethylation. Proc Natl Acad Sci USA. 2012;109(20):7765-7769.

34. Sethi G, Chatterjee S, Rajendran $P$, et al. Inhibition of STAT3 dimerization and acetylation by garcinol suppresses the growth of human hepatocellular carcinoma in vitro and in vivo. Mol Cancer. 2014;13:66. 
A
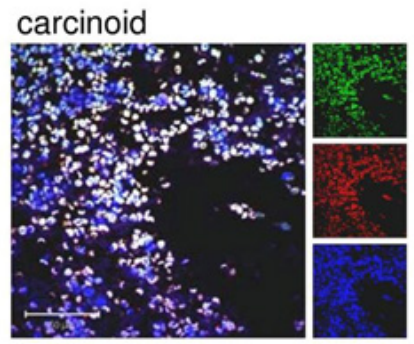

lymphoma

B

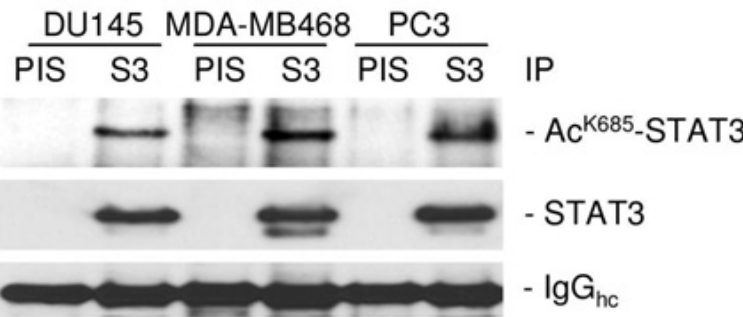

undiff. ca.
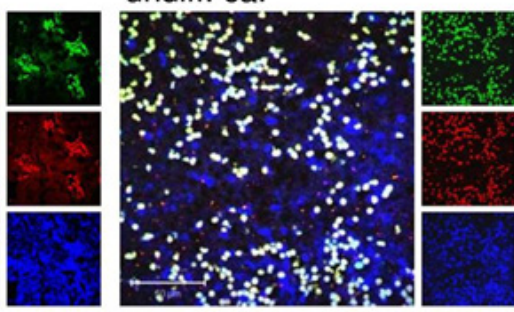

․ AC $\mathrm{C}^{\mathrm{K} 685}-\mathrm{STAT3}$

p p 705 -STAT3

- Hoechst33342

Supplementary Figure SI Frequent STAT3 acetylation in tumor and cancer cell lines.

(A) Tumor sections (carcinoid, lymphoma, undifferentiated cancer) from patients were stained for K685- acetylated and Y705-phosphorylated STAT3 and analyzed by confocal microscopy. Scale bar, $50 \mu \mathrm{m}$.

(B) STAT3 was immunoprecipitated using whole cell lysates from cancer cell lines as indicated and acetylated STAT3 was analyzed in Western blotting. Pre-immune serum (PIS) was used as a control for STAT3 pull down.

A

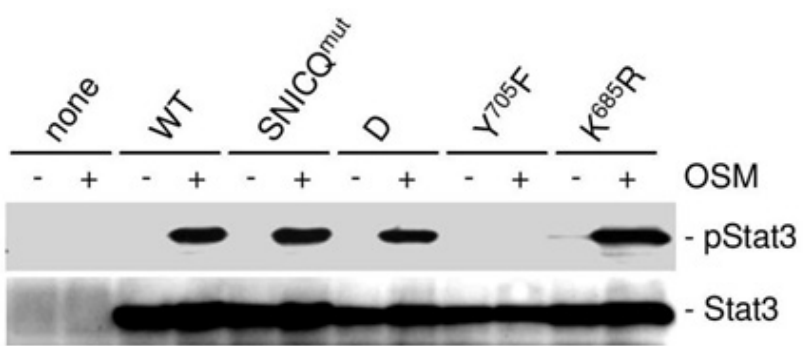

B

Stat3wt

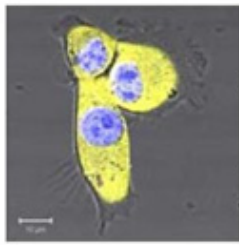

none

OSM

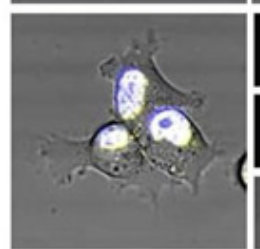

\section{Stat3 $\mathrm{Y705F}$}
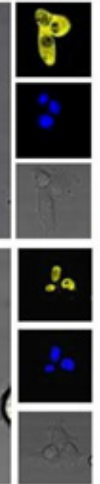
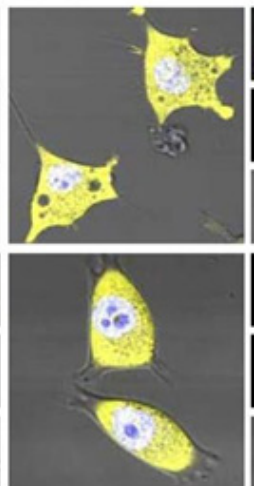

\section{Stat3 ${ }^{K 685 R}$}
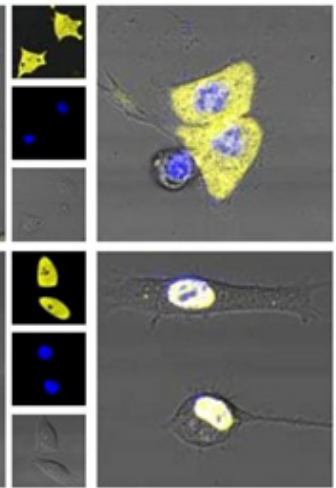

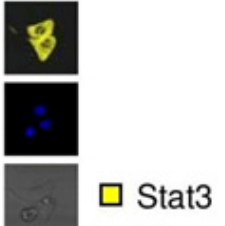

口 Hoechst

- $\square$ DIC

Supplementary Figure S2 Immediate early functional activation of acetylation-deficient STAT3- K685R-YFP. Stat3-YFP constructs as indicated were transiently expressed in mouse $\mathrm{MEF}^{\mathrm{Stat} 3 \Delta \Delta}$ cells and stimulated with $\mathrm{I} 0 \mathrm{ng} / \mathrm{ml}$ OSM for $30 \mathrm{~min}$.

(A) Whole cell lysates were prepared and subjected to Western blot analysis of PY705-Stat3. Stat3-SNICQmut-YFP and Stat3D-YFP represent DNA-binding deficient mutants. Stat3-Y705F-YFP is thought to be dysfunctional in immediate early activation.

(B) Nuclear translocation of Stat3-YFP constructs as indicated was analyzed in fixed cells by confocal microscopy. Single channels of each overlay image are split and shown. Scale bar, $10 \mu \mathrm{m}$. 
A

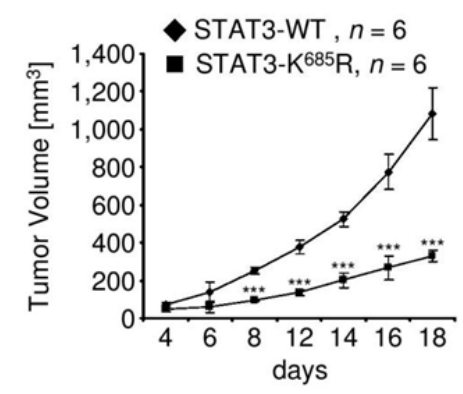

B

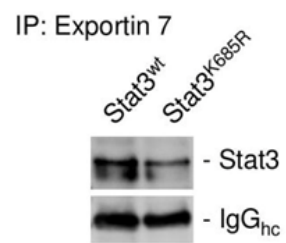

Supplementary Figure S3 Loss of STAT3/exportin 7 interaction upon blocking acetylation in vivo.

(A) Growth kinetics of subcutaneously engrafted tumors expressing STAT3-WT or STAT3- K685R was assessed. SD shown; significance: $* * * P<0.001$

(B) Loss of STAT3/exportin 7 interaction upon blocking lysine acetylation was assessed by immunoprecipitation using tumor homogenates. STAT3 co-precipitation was analyzed in Western blotting.

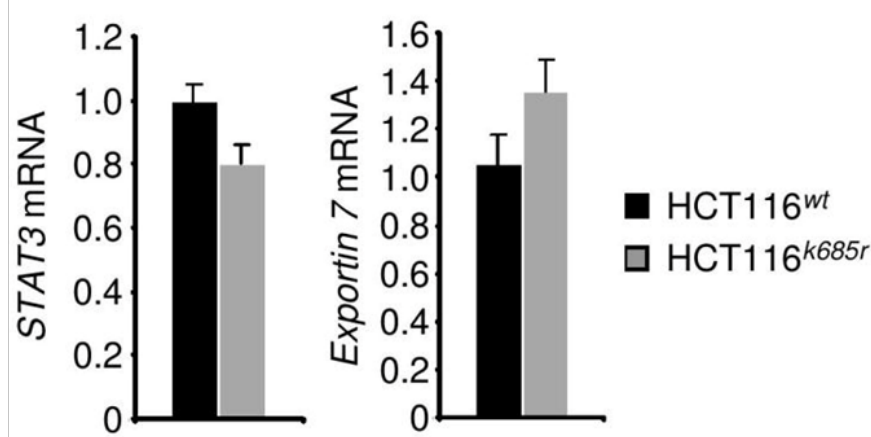

Supplementary Figure S4 Unaffected expression of STAT3 and Exportin 7 mRNA upon inhibition of STAT3 acetylation was determined using human HCT colon carcinoma cells expressing STAT3-WT (HCTII6wt) or STAT3K685R (HCTII6k685r) by RT-PCR. SD shown. 